\title{
Aquarius Active/Passive RFI Environment at L-Band
}

\author{
D. M. Le Vine and P. de Matthaeis
}

\begin{abstract}
Active/Passive instrument combinations (i.e., radiometer and radar) are being developed at L-band for remote sensing of sea surface salinity and soil moisture. Aquarius is already in orbit and SMAP is planned for launch in the Fall of 2014. Aquarius has provided for the first time a simultaneous look at the Radio Frequency Interference (RFI) environment from space for both active and passive instruments. The RFI environment for the radiometer observations is now reasonably well known and examples from Aquarius are presented in this manuscript that show that RFI is an important consideration for the scatterometer as well. In particular, extensive areas of the USA, Europe and Asia exhibit strong RFI in both the radiometer band at 1.41 GHz and in the band at 1.26 GHz employed by the Aquarius scatterometer. Furthermore, in areas such as the USA, where RFI at $1.4 \mathrm{GHz}$ is relatively well controlled, RFI in the scatterometer band maybe the limiting consideration for the operation of combination active/passive instruments.
\end{abstract}

Index Terms-L-band, radio frequency interference, scatterometer.

\section{INTRODUCTION}

A QUARIUS is an L-band active/passive instrument system designed to map sea surface salinity (SSS) from space [1], [2]. The two instruments, scatterometer (active) and radiometer (passive), look at the same surface footprint (i.e., $3 \mathrm{~dB}$ ellipse) at almost the same time. Among the novel results of this mission is the look Aquarius provides of the global Radio Frequency Interference (RFI) environment at L-band for both the radiometer and scatterometer. In active/passive sensor systems such as Aquarius [1] and the Soil Moisture Active/Passive (SMAP) mission [3], the sensors are required to operate together looking at the same pixels at the same time to provide complementary information (to reduce the effect of roughness in the case of Aquarius and to improve resolution in the case of SMAP). Hence, although the sources of RFI may be different for the two instruments, RFI that disables either can compromise the science product. The objective of this letter is to present an initial look at the RFI environment as seen in both the active and passive bands by Aquarius with emphasis on RFI in the active band (scatterometer).

\section{AQUARIUS}

Aquarius consists of three L-band radiometers $(1.41 \mathrm{GHz})$ and an L-band scatterometer $(1.26 \mathrm{GHz})$ designed to work together to map sea surface salinity. The two instruments share

Manuscript received October 25, 2013; revised January 22, 2014; accepted February 7, 2014. Date of publication April 14, 2014; date of current version May 12, 2014.

The authors are with the NASA Goddard Space Flight Center, Greenbelt, MD 20771 USA.

Digital Object Identifier 10.1109/LGRS.2014.2307794 the antenna system which is comprised of three feed horns and an offset fed reflector [1]. The scatterometer shares the feed horns with the radiometers and is designed to have the same boresight and approximately the same $3 \mathrm{~dB}$ footprint ellipse on the surface. The scatterometer measures VV, HV, VH and $\mathrm{HH}$ alternating each transmit-receive pair with a receiveonly observation which is used to establish the noise floor [1]. Aquarius maps the globe every 7 days in an exact-repeat orbit (i.e., returning along the same path with the same look every 7 days). This has an advantage for remote sensing of a parameter such as sea surface salinity which changes relatively slowly [2]; however, it limits the information one can obtain about RFI. In particular, it prevents locating sources of RFI using techniques based on repeat passes with slightly different perspective as has been successfully employed by the Soil Moisture and Ocean Salinity (SMOS) mission [4].

\section{RFI DETECTION}

The approach adopted for RFI detection and mitigation in Aquarius was to sample rapidly so that samples corrupted by RFI could be identified and removed without losing data for the entire pixel. The fundamental sample window is $10 \mathrm{~ms}$. The scatterometer transmits during the first $\mathrm{ms}$ of this window during which the radiometer is blanked, and then both radiometer and scatterometer receive during the remaining $9 \mathrm{~ms}$. The scatterometer receive window (i.e., the time during which the beam is filled) is $2-3 \mathrm{~s}$. The complete cycle of radiometer data acquisition is a sequence of 12 such $10 \mathrm{~ms}$ intervals. The radiometers observe the scene during the first 7 of the 12 intervals, followed by 5 intervals of internal calibration. The complete scatterometer cycle is $180 \mathrm{~ms}$ during which the scatterometer cycles between all polarization combinations and the three feed horns. (See [1, Fig. 7] for a timing diagram.)

\section{A. Radiometer}

The Aquarius radiometer RFI detection algorithm is an adaptation of an algorithm designed to detect pulsed interference such as expected from a radar [5]. The algorithm is a timedomain glitch detector in the sense that it compares a sample under test with a local mean obtained from neighboring samples, and it rejects the sample if it deviates too much from the local mean. The process begins by forming a string of the raw data samples (one each $10 \mathrm{~ms}$ ) with zeros applied during the intervals of internal calibration. Each string consists of 7 data samples and 5 zeros (the calibration samples) in a regular sequence which is repeated 12 times. This $1.44 \mathrm{~s}$ of data (i.e., $12 \times 120 \mathrm{~ms}$ ) is the basic data block used after RFI is removed for processing Aquarius data to salinity. The test for RFI is 
applied to each non-zero sample in this string and consists of the following steps [5], [6].

1) Computation of a "dirty" mean using all non-zero samples within $\pm \mathrm{W}_{m}$ steps of the sample under consideration. $\mathrm{W}_{m}=20$ steps in the current version of the algorithm.

2) All samples which exceed the "dirty" mean by a threshold, $\mathrm{T}_{\mathrm{m}}$, are excluded and a new, "clean" mean, is computed.

3) Samples are flagged as RFI if they differ from the "clean" mean by more than a second threshold, $\mathrm{T}_{\mathrm{d}}$. In addition, samples within $\pm \mathrm{W}_{d}$ steps on either side of any sample flagged as RFI (counting zeros) are considered tainted and also flagged as RFI. $\mathrm{W}_{d}=2$ in the current algorithm.

The thresholds in steps 2 and 3 are set based on the noise of the radiometer output (i.e., NEDT estimated after launch). The threshold in step 2 is 1.5 NEDT and in step 3 it is 4 NEDT. All the parameters of the algorithm are adjustable and the values currently being used are given in [6, Table I] where a more detailed description of the algorithm can also be found.

\section{B. Scatterometer}

The scatterometer algorithm consists of on-board processing of the data in the receive-only windows followed by processing on the ground of the data in all the windows using an algorithm similar to that employed by the radiometer [7], [8].

1) Onboard RFI Detection: On-board detection is employed to take advantage of the high time resolution of the raw digital sampling before the data is integrated for transmission to the ground. This is done only in the receive-only windows. Assuming that, in the absence of RFI and radar return, the distribution in the receive-only window is Gaussian (e.g., due to instrument and ambient thermal noise), a threshold is set on the tails of the distribution to identify RFI. A power level threshold and a number of digital counts that exceed this level is specified. If the digital hardware detects the specified number of counts exceeding the power threshold an RFI flag is set. In addition, the data in the transmit-receive windows before and after this window are also flagged as RFI.

As currently implemented, the default onboard power threshold is $14 \mathrm{mV} \mathrm{rms}$ which corresponds to a received power level at the antenna of $-120 \mathrm{dBW}$ and is approximately three-sigma above the receiver noise floor level [7]. Since the analog-todigital converter (ADC) samples the scatterometer signal at $16 \mathrm{MHz}$, about 100 ADC samples $(0.3 \%)$ should exceed this power level in a $2 \mathrm{~ms}$ range gate under ambient conditions (no RFI). The most likely RFI sources (e.g., air traffic control radar) have pulse lengths of about $100 \mu \mathrm{s}$ corresponding to about 1600 ADC samples when the threshold is exceeded. The default trigger count was set to 400 ADC samples. An obvious limitation on this approach is that the algorithm depends on the assumed characteristic of the RFI. It is possible to change the default power and trigger count thresholds with a command from the ground, but the current algorithm uses the default values.

Once Aquarius was in orbit, it was observed that RFI was escaping detection by the on-board RFI flagging technique.
Strong RFI was appearing in transmit-receive "echo" windows but not in adjacent receive-only "noise" windows. This is most likely due to "frequency hopping" by the ground transmitters, where radar pulses a few milliseconds apart can differ in frequency, with one pulse lying within the Aquarius $5 \mathrm{MHz}$ receive band and the adjacent pulses lying outside of this band [7]. To address this, an independent ground-based algorithm was implemented.

2) Ground-Based RFI Detection: The ground-based RFI detection technique is an outlier detection algorithm similar to that developed for the radiometer. RFI flags from the on-board algorithm are retained, and scans for additional RFI are made using the following steps:

1) Receive-only measurements that exceed a threshold of $-33 \mathrm{dBm}$ are flagged as RFI. This is an attempt to identify RFI that might have escaped detection because its pulse characteristics do not fit the assumptions above. When the correlated noise diode (CND, one of the internal calibration sources [1]) is turned on, a higher threshold of $-31 \mathrm{dBm}$ is used for the V-pol channel which can (by design) see the CND.

2) The median and the standard deviation is computed using 7 data samples on each side of the sample under test and excluding the test sample. This is done for all measurements (i.e., transmit-receive and receive-only and all polarization combinations).

3) Outliers are flagged. If the absolute value of the difference between the sample under test and the associated median value exceeds $\mathrm{N}$ standard deviations, the test point is flagged as RFI. A limit is set on the maximum standard deviation allowed for this test because some regions are so heavily corrupted by RFI that the statistics are corrupted and reflect the level of RFI rather than the signal from the surface. In the current implementation, the maximum standard deviation is $0.001 \mathrm{~mW}$ and $\mathrm{N}=$ 6 for the transmit-receive channels and $\mathrm{N}=5$ for the receive-only channels.

4) The points flagged as RFI are replaced with their corresponding median values and then step 2 above is repeated. New values are computed for the median and standard deviation for all data. The objective is to make a second pass through the data looking for more subtle RFI.

5) Step 3 above is repeated. Outliers are flagged as RFI. The same thresholds are used as in step 3.

\section{EXAMPLES}

\section{A. Radiometer}

Fig. 1 illustrates the global distribution of RFI as measured by the Aquarius radiometers. The percentage of samples flagged as RFI is shown for one year (2012) and is representative of the data collected to date. Some obvious features are the strong correlation of high percentages of RFI with the population centers in South America and Africa and the persistent RFI in Eastern Europe and China. By comparison, RFI over the ocean is relatively infrequent except in coastal regions of the North Atlantic and in the Western Pacific near 


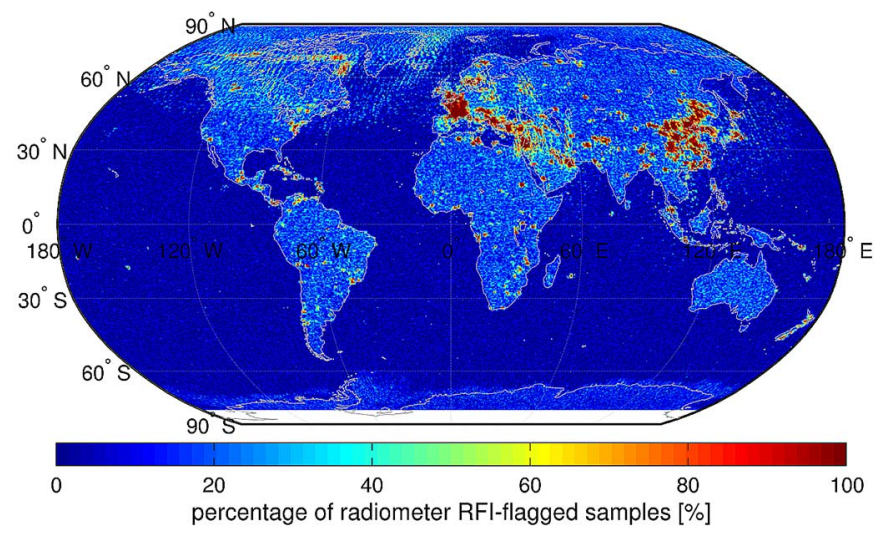

Fig. 1. Percentage of Aquarius radiometer samples identified as RFI for the year, January 1 to December 31,2012 . The percentage at each point is the average of both polarizations (i.e., $[\mathrm{V}+\mathrm{H}] / 2$ ).

the coast of China. This most likely is caused by strong sources of RFI elsewhere (i.e., on land) entering through the antenna sidelobes. This conclusion is supported by comparing ascending and descending passes where differences in the antenna pattern and orientation create differences in the pattern of the observed RFI which would be unlikely if the RFI originated near boresight (e.g., see [6, Figs. 5, 6]).

The Aquarius radiometers operate in the protected band at $1.413 \mathrm{GHz}$ where in principle there should be no RFI. However, RFI is detected for several reasons, among them the existence of violators of the rules either illegally transmitting in this band or not controlling spurious out-of-band radiation. Another reason is the fact that the Aquarius passband filters do not completely exclude strong signals in the nearby spectrum. The roll-off rate of the filters is a compromise between obtaining maximum bandwidth to reduce radiometric noise (i.e., NEDT) and the need for RFI suppression. Since the primary application of Aquarius is over the ocean for the measurement of salinity, a decision was made to design to maximize bandwidth at the cost of some out-of-band rejection. SMOS made a different decision and has sharper filter skirts near the band edge. Some consequences of this difference can be seen in the RFI in Fig. 1 associated with the DEW line radars in Canada (the horizontal line at about $80^{\circ}$ north latitude) and the very high percentage of RFI (red area) over France. These are persistent features in Aquarius but are not observed in SMOS. Additional examples illustrating the characteristics of the RFI observed by Aquarius and SMOS can be found in [6], [9] and current examples of RFI can be found in [10] for SMOS and [11] for Aquarius. The focus in this letter is on the global distribution of scatterometer RFI.

\section{B. Scatterometer}

Among the new results from Aquarius is a first look at the global distribution of RFI as encountered by the scatterometer. The scatterometer operates in a band at 1.24-1.30 GHz set aside for active remote sensing from space (i.e., "Earth ExplorationSatellite" active) but shared with radionavigation [12]. The later includes civilian and military air traffic control and surveillance radar (e.g., FPS-117; ARSR-1 and 2; FPS-124; etc).

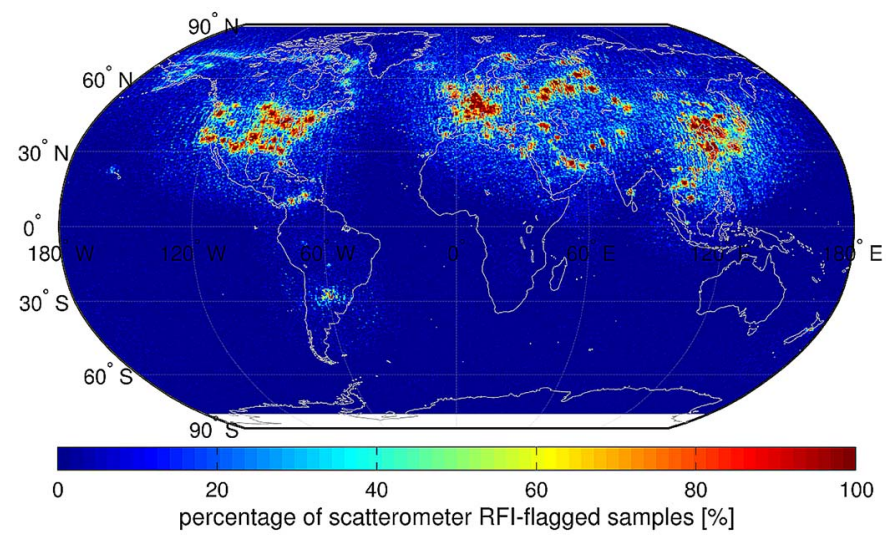

Fig. 2. Percentage of scatterometer samples identified as RFI. The percentage at each point is the average for both polarizations (i.e., $[\mathrm{V}+\mathrm{H}] / 2$ ) and averaged over the year, January 1 to December 31, 2012.

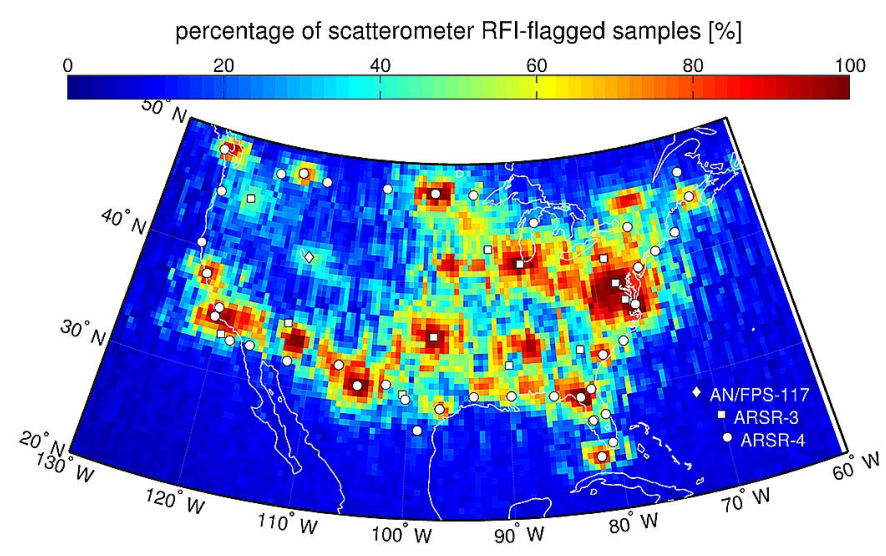

Fig. 3. Scatterometer RFI map for the USA for the year 2012 with the location of L-band radar supperimposed on the map. The data is the same as in Fig. 2 with an expanded scale to show the continental USA in more detail.

Fig. 2 shows the global distribution of RFI as seen by the Aquarius scatterometer. The percentage of samples flagged as RFI is shown averaged over the one year (January 1December 31, 2012); however, the patterns of RFI are very stable changing little from week to week. Some well-known radars can be seen. For example, the North Warning System (NWS) - formerly DEW line_-radars around Alaska, crossing Canada, and then south following the east coast of Canada are clearly evident (light blue line of spots between 60-80 north latitude). The mainland United States which is relatively free of RFI at $1.413 \mathrm{GHz}$ (Fig. 1), has large areas of very persistent RFI in the case of the scatterometer (red in Fig. 2). These are well correlated with population centers and other locations where one would expect air traffic control radar. For example, Fig. 3 shows the scatterometer RFI map for the continental USA together with the location of known radar (ARSR-3, ARSR-4, and AN/FPS-117) which transmit in the Aquarius scatterometer band. There is a strong correlation of the location of intense RFI (red) and the symbols indicating sites of radar. There are some obvious exceptions (e.g., in the vicinity of Phoenix, AZ and Memphis, TN) but these are likely the result of omissions in the radar database which was compiled in 2004 and only included non-classified locations [8]. 


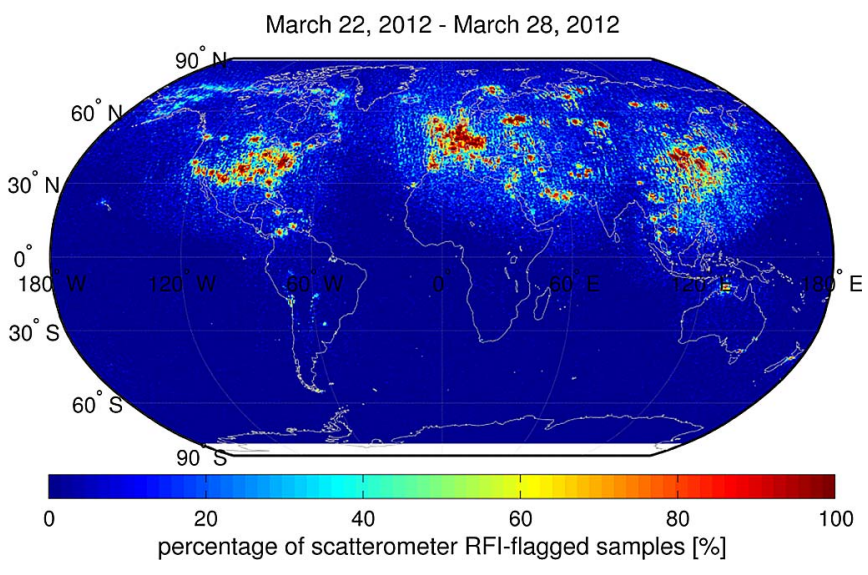

Fig. 4. Percentage of Aquarius scatterometer samples identified as RFI during one 7-day cycle: March 22-28, 2012. The data are the average of measurements at vertical and horizontal polarization.

The RFI map in Fig. 2 shows one year of data and the average of observations at vertical and horizontal polarization. The general pattern changes very little with time or polarization. For example, Fig. 4 shows the RFI map for one 7-day cycle of Aquarius scatterometer measurements (Aquarius maps the globe once every 7 days). This example is for March 22-28, 2012 but there is little change from week to week and the general pattern is similar to the year average shown in Fig. 2. In the areas of high likelihood of encountering RFI (red) there is very little dependence on polarization or orientation (i.e., ascending or descending orbits). However, there is some variation in the lower percentage "halos" surrounding the high percentage (red) sources. The halos are most likely the result of strong signals from radar on land, probably in the vicinity of the red areas and entering through the antenna sidelobes. The same phenomena occurs in the case of radiometer RFI and is somewhat more obvious in the case of the scatterometer (Fig. 2) where it is relatively certain that the sources are ATC radar. This is illustrated in Fig. 5 which shows the same data as in Fig. 2 separated into ascending (top) and descending (bottom) orbits. The pattern of red remains largely unchanged, but there are noticeable changes in the light blue in the Pacific off the coast of China and along the west coast of the USA and Europe. For more information on the orientation of the antenna patterns see [1, Fig. 3] and [6, Fig. 1].

Fig. 6 shows the difference in the scatterometer signal (radar cross section, $\sigma^{\circ}$ ) before and after RFI mitigation (removal of samples flagged as RFI). Assuming that the RFI algorithm is reliable, this should provide an indication of the relative amplitude of the RFI. The patterns in Fig. 6 and Fig. 2 (frequency of occurrence) are very similar. For low levels of RFI (frequency $<50 \%$ and level $<5 \mathrm{~dB}$ ) there is a strong correlation between level and frequency of occurrence (these numbers are based on calculations not shown here for lack of space). This is the range indicated by light blue in both figures and includes the halo surrounding the red areas and extending into the ocean where one does not expect to find physical sources of radiation. One explanation is that the probability of detection depends on the strength of the signal up to some level after which the probability of detection depends only on the likelihood of the
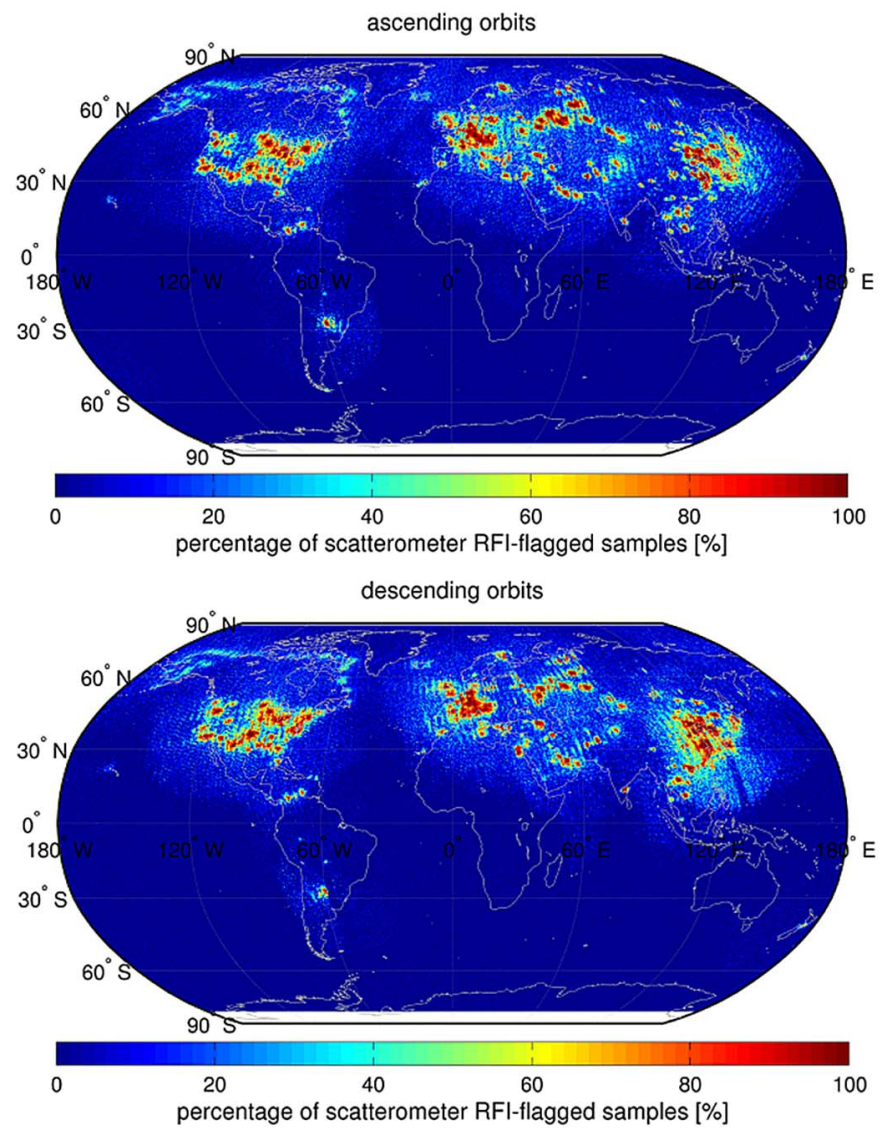

Fig. 5. Comparison of RFI in ascending (top) and descending (bottom) orbits. Data is for January 1 to December 31, 2012 and an average of data at vertical and horizontal polarization.

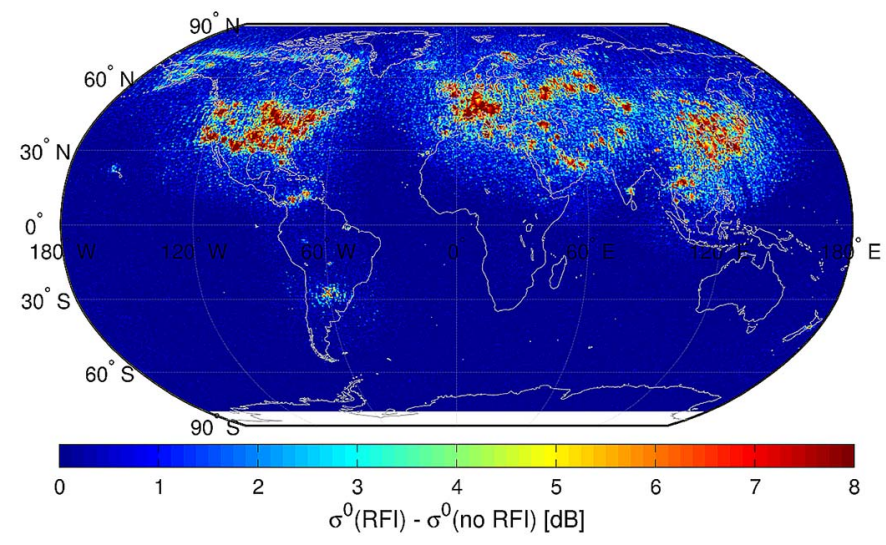

Fig. 6. Amplitude of the scatterometer RFI. The difference between the scatterometer signal before and after RFI is removed, averaged over polarization, and for the year, January 1 to December 31, 2012

source transmitting (i.e., not on amplitude) when the satellite passes over. One would expect the latter case when the antenna boresight passes over the source of RFI and no protection is provided by the antenna.

\section{DiscusSION}

It is useful to compare areas contaminated by RFI for the radiometer and for the scatterometer. This is important for combined active/passive instrument systems such as Aquarius 


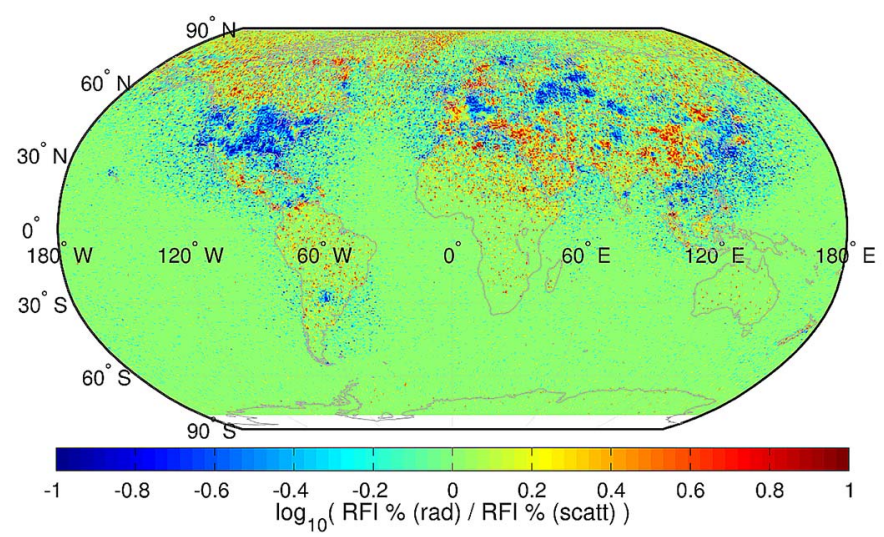

Fig. 7. Logarithmic ratio of percentage of radiometer samples identified as RFI to the percentage of scatterometer samples identified as RFI for the year, January 1 to December 31, 2012.

and SMAP which rely on both sensors having RFI-free measurements at the same place and time to meet their measurement objectives [2], [3]. Fig. 7 plots on a logarithmic scale the ratio of the percentage of samples flagged as RFI for the radiometer to the percentage flagged as RFI in the case of the scatterometer. Red indicates that RFI is more important for the radiometer and blue indicates that RFI is more important for the scatterometer. Comparing with Fig. 2 (frequency of occurrence for the scatterometer) it is clear that the radar sites (red in Fig. 2 and blue in Fig. 7) dominate in the case of the scatterometer but the halo areas (e.g., around the USA and in the Pacific Ocean along the east coast of Asia) are also significant. The areas of red (dominant problem for the radiometer) are also clearly evident in Fig. 1. Over the ocean, except near the coast of the USA, Europe, and Asia, RFI is much less of a problem than over land. Over the USA RFI is more of a problem for the scatterometer, while over eastern Asia RFI is more of a problem for the radiometer (because out of band RFI is poorly controlled as indicated in Fig. 1). In Europe and the Middle East, RFI is a problem for both the radiometer and scatterometer.

Finally, it is to be noted that the examples presented here (e.g., Figs. 1, 2) illustrate "detected" RFI: That is, the output of the algorithms. The maps correctly demonstrate the distribution of RFI, but the algorithms are imperfect and the numbers include an element of false alarms and missed detection. They also do not necessarily represent the true location of RFI because strong signal may enter via the sidelobes (e.g., the halos off the west coast of Asia). Improving the algorithms is an ongoing area of work. SMAP will implement the next generation of RFI detection for radiometers at L-band and comparison of the SMOS, Aquarius and SMAP algorithms will provide insight into the optimum approach to this problem. Also, in the case of the SMAP scatterometer, the ability to shift frequency has been added to help avoid RFI. Hopefully, this will help, but the problem of operating in a shared band with strong sources which also can change frequency is still likely to be a significant challenge.

\section{REFERENCES}

[1] D. M. Le Vine, G. S. E. Lagerloef, F. R. Colomb, S. H. Yueh, and F. A. Pellerano, "Aquarius: An instrument to monitor sea surface salinity from space," IEEE Trans. Geosci. Remote Sens., vol. 45, no. 7, pp. 2040 2050, Jul. 2007.

[2] G. S. E Lagerloef, F. Colomb, D. Le Vine, F. Wentz, S. Yueh, C. Ruf, J. Lilly, J. Gunn, Y. Chao, A. deCharon, G. Feldman, and C. Swift, "The Aquarius/SAC-D mission: Designed to meet the salinity remote sensing challenge," Oceanography, vol. 21, no. 1, pp. 68-81, Mar. 2008.

[3] D. Entakhabi, E. G. Njoku, P. E. O'Neill, K. H. Kellogg, W. T. Crow, W. N. Edelstein, J. K. Entin, S. D. Goodman, T. J. Jackson, J. Johnson, J. Kimball, J. R. Piepmeier, R. D. Koster, N. Martin, K. C. McDonald, M. Moghaddam, S. Moran, R. Reichle, J.-C. Shi, M. W. Spencer, S. W. Thurman, L. Tsang, and J. Van Zyl, "The soil moisture active passive (SMAP) mission," Proc. IEEE, vol. 98, no. 5, pp. 704-716, May 2010.

[4] R. Oliva, E. Daganzo, Y. H. Kerr, S. Mecklenburg, S. Nieto, P. Richaume, and C. Gruhier, "SMOS radio frequency interference scenario: Status and actions taken to improve the RFI environment in the $1400-1427-\mathrm{MHz}$ passive band," IEEE Trans. Geosci. Remote Sens., vol. 50, no. 5, pp. 1427-1439, May 2012.

[5] C. Ruf and S. Misra, "Detection of radio-frequency interference for the Aquarius radiometer," IEEE Trans. Geosci Remote Sens., vol. 46, no. 10, pp. 3123-3128, Oct. 2008.

[6] D. M. Le Vine, P. de Matthaeis, C. Ruf, and D. Chen, "Aquarius RFI detection and mitigation algorithm and examples," IEEE Trans. Geosci. Remote Sens., vol. 52, no. 8, pp. 4574-4584, Aug. 2014.

[7] S. Yueh, A. Fore, A. Freedman, M. J. Chaubell, W. Tang, and G. Neumann, Aquarius Scatterometer Algorithm Theoretical Basis Document, (Section 8), Mar. 15, 2012. [Online]. Available: ftp://podaac-ftp.jpl. nasa.gov/allData/aquarius/docs/v2/AQ-014-PS-0019_AquariusATBD_ scatterometer.pdf

[8] A. P. Freedman, J. R. Piepmeier, M. A. Fischman, D. A. Watters, and M. W. Spencer, "The detection and mitigation of RFI with the aquarius Lband scatterometer," in Proc. Int. Geosci. Remote Sens. Symp., Aug. 2008, vol. II, pp. II-319-II-322.

[9] M. Aksoy and J. T. Johnson, "A comparative analysis of low-level radio frequency interference in SMOS and aquarius microwave radiometer measurements," IEEE Trans. Geosci. Remote Sens., vol. 51, no. 10, pp. $4983-$ 4992, Oct. 2013.

[10] [Online]. Available: http://www.cesbio.ups-tlse.fr/SMOS_blog/?tag=rfi

[11] [Online]. Available: http://aquarius.umaine.edu/cgi/data.htm

[12] Federal Communications Commission (FCC), Table of Frequency Allocations, April 2013. [Online]. Available: http://transition.fcc.gov/oet/ spectrum/table/fcctable.pdf 